\title{
Considerations before orthodontic camouflage treatment in skeletal class III malocclusion
}

\author{
Marcella Budhiawan*, Haru Setyo Anggani** \\ *Student of Orthodontic Specialist Programme Faculty of Dentistry Universitas Indonesia \\ **Department of Orthodontic Faculty of Dentistry Universitas Indonesia
}

\section{ABSTRACT}

Skeletal Class III malocclusions are caused by maxillary deficiency, mandibular protrusion, or a combination of the two. This patient in this case may have a sunken in face, strong chin appearance. Most persons with Class III malocclusions, which is a dentofacial deformity, show combinations of skeletal and dentoalveolar components. Orthodontic therapy is usually aimed at compensating for the underlying mild-moderate skeletal Class III discrepancy and patients with severe skeletal Class III discrepancies require a combination of orthodontic treatment and orthognatic surgery to correct the underlying skeletal pattern. By considering many factors, the orthodontic treatment can be done on mild to severe skeletal Class III. These factors are facial profile, dental relationship and skeletal pattern. Those factors should be considered a starting point in making treatment decision. They gives the limitation of orthodontic treatment in terms of whether the occlusion could be corrected, or whether the deformity could be camouflage.

Key words: Skeletal Class III malocclusions, camouflage treatment

\section{INTRODUCTION}

Class III malocclusion is one of the most difficult anomalies to understand. ${ }^{1}$ Class III malocclusion can be defined as a skeletal facial deformity characterized by a forward mandibular position with respect to the cranial base and/or maxilla. This malocclusion is characterized by the position of the mandible projecting too far forwards than the maxilla. Most orthodontist are familiar with the difficulties associated with the treatment of Class III malocclusion. Characteristics of skeletal Class III malocclusion could be summarized as the following overdeveloped mandible, underdeveloped maxilla, or a combination of both. Dentoalveolar relationship shows Class III molar relationship, proclined maxillary incisors and retroclined mandibular incisors with anterior and/or posterior crossbite. ${ }^{2,3}$ The patient in this case may have a sunken in face, and usually they face the psychosocial problems. ${ }^{4-6}$

The Class III malocclusion is a hereditarily conditioned. The frequency of this malocclusion is about $1-3 \%$, depending on age and geographic variation. ${ }^{7}$ In Asian societies, the frequency of Class III malocclusion is higher, because of a large percentage of patients with maxillary deficiency. ${ }^{8}$

The choice of treatment of skeletal Class III malocclusion depends on the age and the severity of the malocclusion. When the permanent 
dentition has established, orthodontic therapy usually aimed at compensating for the underlying mild-moderate Class III skeletal discrepancy. In contrast, adolescent and non-growing patient with severe Class III skeletal discrepancies require a combination of orthodontic treatment and orthognathic surgery to correct the underlying skeletal pattern. For patients reluctant to undergo surgery or who are satisfied with their facial appearance, an alternative is to treat with dentoalveolar compensation without correcting the underlying skeletal deformity. ${ }^{1,9}$ To achieve the goals of camouflage, we have to know the limits of dentoalveolar compensation and the mechanics which provide significant dentoalveolar changes without unfavourable side effects. In undertaking the decision to treat such a severe Class III condition through dentoalveolar compensation, the clinicians must be very careful, because it is more difficult to obtain acceptable esthetics. If not, the final result is only the compromise. ${ }^{9}$

Although the frequence of this malocclusion is rather small, but it's been sugested that the treatment should be done to obtain satisfactory dental and facial esthetic. Class III camouflage is more difficult than the other malocclusion, so the clinicians must be very careful. As mentioned above, an orthodontist should know the considerations before doing the camouflage orthodontic treatment.

\section{CLASS III MALOCCLUSION}

Class III malocclusion is one of the most difficult anomalies to understand. Studies conducted to identify the etiological features of a Class III malocclusion showed that the deformity is not restricted to the jaws but involves the total craniofacial complex. Most persons with Class III malocclusions shows combinations of skeletal and dentoalveolar components. ${ }^{1}$

Class III malocclusion can be defined as a skeletal facial deformity characterized by a forward mandiblular position, with respect to the cranial base and/or maxilla. This facial dysplasia can be classified into mandibular prognathism, maxillary retrognathism, or a combination of both. . $^{3,6,8,10,11,13}$ Class III anteroposterior skeletal imbalances are usually attributable to one or more of the following component variables, the mandible may be too large relative to the maxilla, the maxilla may be too small relative to the mandible, the maxilla may be retropositioned relative to the mandible, the mandible may be positioned too far forward relative to the maxilla a forward rotation of the mandible relative to the cranium will cause the chin point to move into a horizontally more protrusive position. A prognathous mandible may thereby result, together with a reduction in lower anterior facial height. ${ }^{10}$

Component variables have been studied frequently by means of cephalometric roentgenography. According to Jacobson ${ }^{10}$, Class III malocclusion has been divided into two basic morphologic types the divergent and the convergent types (Fig. 1\& 2). Typically characteristic features of the divergent Class III pattern are palatal, occlusal, and mandibular planes which diverge, an obtuse gonial angle, and an anterior open-bite in extreme cases. The convergent Class III patterns features palatal, occlusal, and mandibular planes which tend toward parallelism, an acute gonial angle, and a deep anterior overbite. ${ }^{7}$

Sugawara ${ }^{11}$ divided Class III malocclusion vertically into three basic types depending on the vertical disporpotions: long, average, and short face. Schudy ${ }^{10}$ introduced the terms hyperdivergent and hypodivergent.

The nature of craniofacial form is a composite assembly pattern of the various skeletal components in this region. The profile of the face is thus dependent upon various combinations of these craniofacial skeletal components in a horizontal as well as a vertical dimension. Balanced facial profiles are achieved only when there exists a favorable combination of these components in the both dimensions. ${ }^{3,10}$

For assesment of the balanced facial profile, we have to know the determination of the resultant profile relationship of the upper and lower jaws to the cranium, regardless of any variables within the skeletal composite. ${ }^{10}$

\section{Range of prognathism}

Jacobson $^{10}$ found the most significant difference between the Class III malocclusion and the normal occlusion sample was the ANB angles $\left(<2^{\circ}\right)$. These marked ANB angle differences reflect a forward positioning of the Class III mandible relative to the anterior cranial base. This 


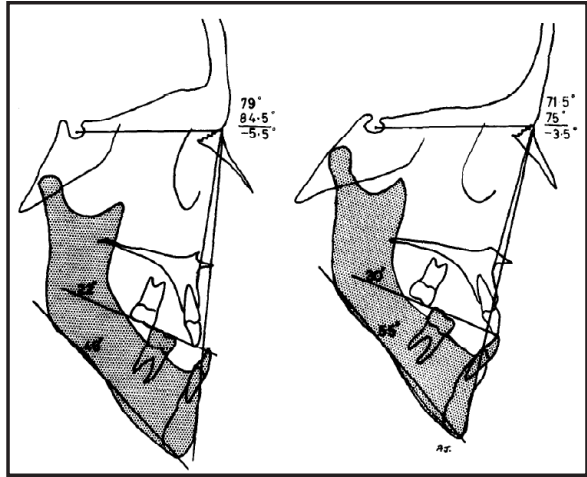

Figure 1. Divergent types of Class III craniofacial skeletal patterns. ${ }^{10}$

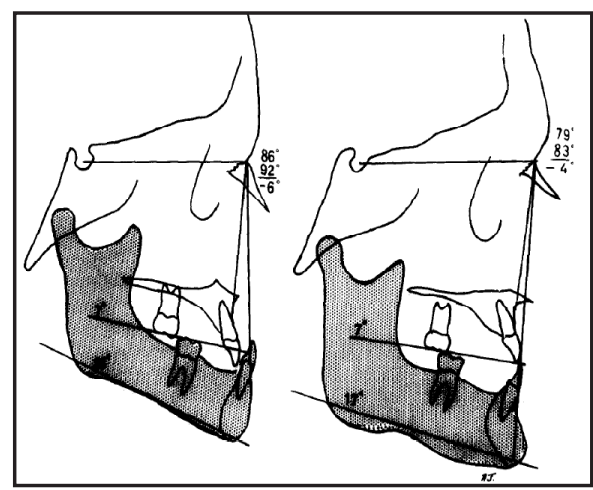

Figure 2. Convergent types of Class III craniofacial skeletal patterns. ${ }^{10}$

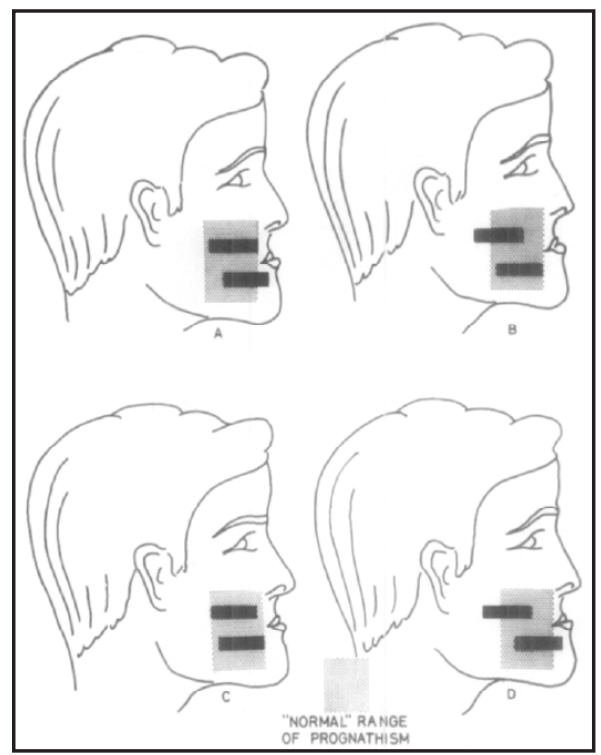

Figure 3. Variation in the Class III profile. ${ }^{10}$

relationship was recorded by the mean SNB angle measurements, which are significantly larger in the Class III sample than in the normal occlusion. A comparative maxillary retrusion accentuates the typical Class III profile. This retropositioning may be partly associated with the shorter anterior cranial base found in the Class III sample, but it is also a result of a distal location of the maxilla relative to the anterior cranial base, as determined by measuring the SNA angles which are slightly but neverthless significantly smaller in the Class III sample. First, we assessed the relationship of the upper and lower jaws to the carnial base. In males the normal range prognathism in the maxilla extended from 79.0 to $87.0^{\circ}$ (SNA mean of normal occlusion being $83.20^{\circ} \pm 4.09^{\circ}$ ) and in the mandible from $77.0^{\circ}-85.0^{\circ}\left(\mathrm{SNB}=81.11^{\circ} \pm 4.03^{\circ}\right)$. In females, the normal range prognathism in the maxilla extended from $78.5^{\circ}-86.0^{\circ}$ (SNA $=$ $\left.82.26^{\circ} \pm 3.83^{\circ}\right)$ and in the mandible from 76.0 $83.5^{\circ}\left(\mathrm{SNB}=79.66 \pm 3.88^{\circ}\right)$.

Jacobson ${ }^{10}$ divided Class III malocclusion into the following categories (Fig. 3). Group A: Maxilla within the normal range of prognathism, mandible beyond the normal range; Group B: Maxilla below the normal range of prognathism, mandible within the normal range; Group C: Maxilla and mandible within the normal range of prognathism; Group D: Maxilla below the normal range of prognathism, mandible beyond the normal range of prognathism; Group E: Maxilla and mandible beyond normal range of prognathism; Group F: Maxilla and mandible below normal range of prognathism; Group G: Maxilla within normal range prognathism, mandible below normal range.

\section{Skeletal morphology}

Characteristic of Class III malocclusion is a forward position of mandible relative to the maxilla. ${ }^{8,14}$ In some cases the lower jaw can grow out too far in front of the upper jaw, this is called mandibular prognathism. The other condition commonly seen in under bite patients is when maxilla or top jaw has not properly developed. This condition is called mid-face deficiency. ${ }^{15}$ General morphologic differences in the mandibles of persons with Class III malocclusions and normal occlusions included significantly greater mean total effective lengths of the Class III mandibles. ${ }^{10}$

Class III patients showed a strong tendency toward middle face deficiency, this was indicated by four angular measurements which were significantly smaller: sella-nasion-ANS; SNA; sella-nasion-prosthion and posteroinferior angles formed by $\mathrm{N}-\mathrm{A}$ and the Frankfort plane. ${ }^{16}$ Maxillary 
retrusion may be partly associated with the shorter anterior cranial base, and also a result of a distal location of the maxilla relative to the anterior cranial base. ${ }^{10}$

In Class III malocclusion (mandibular prognathism or mid-face deficiency) presents a minus angle of convexity (formed by N-A and A$\mathrm{Pg}) .{ }^{12}$ Malar eminence deficiency and a flatness of the orbital rim, often is present in patients with mid-face deficiency (as seen on cephalometric roentgenography). ${ }^{17,18}$ Mandibular length (Co-Gn) in class III malocclusion was significant longer than in normal occlusion. ${ }^{14}$

Characteristic of Class III malocclusion is the prominence of the chin that caused the lower lip protruded and the facial esthetic may result in less. Clinician should evaluate the primary problem of the mandibular prognathism. Is it because of overgrowth of the chin or overgrowth of the mandible. ${ }^{13}$ Chin projection is determined by the amount of anteroposterior bony projection of anterior, inferior border of the mandible. $\mathrm{NB}-\mathrm{Pg}$ is the cephalometric measurement most orthodontist refer to as chin projection. ${ }^{19}$

Gonial angle in Class III malocclusion is more obtuse than in normal occlusion. ${ }^{10,16,18}$ The obtuseness of the gonial angle in Class III affected the lower border of the mandible which was more steeply inclined then in the normal. This was shown by the significantly greater angle formed by the Gonion-Gnathion plane with the S-N, Frankfort, palatal, and occlusal plane. ${ }^{16}$

The anterior facial height in Class III malocclusions is significantly greater than normal occlusion. The mean posterior facial heights are not significantly different from the normal occlusion. The anterior facial height is associated with the predominantly divergent type of Class III pattern or vertical facial pattern. ${ }^{16}$

Bjork theorized that if the saddle angle, nasion-sella-artikular, became smaller, the temporomandibular joint would be displaced forward which, in turn, would affect the mandible and cause an increase in the amount of prognathism. ${ }^{16,18}$ Fig. 4 illustrated lateral cephalometri that showed skeletal Class III malocclusion. ${ }^{12}$

\section{Dental morphology}

In Class III malocclusion maxillary anterior teeth are protrusive and facially inclined and the maxillary dental arch takes a $\mathrm{V}$ shape in the anterior aspect. ${ }^{17}$ If there was a missing teeth or the eruption abnormality, such as ectopic canines and palatal eruption of lateral incisors, could cause maxillary deficiency and crowding of the teeth (Fig. 5). ${ }^{20}$

The mandibular anterior teeth may be upright in relation to the basal supporting bone of the mandible, or they may be inclined lingually. The mandibular incisors may be crowded. ${ }^{17}$ In generally, this malocclusion showed a reversed horizontal overlap in incisor area. ${ }^{21}$ But could also have an edge to edge incisor relationship or normal. ${ }^{11}$

Nanda $^{6}$ reported that occlusion could depend on each skeletal facial type. Typically characteristic features of a long face type is an anterior open bite. In a short face type represents a deep bite (Fig. 6). ${ }^{11}$ The most commonly cited feature of mandibular excess is the mesial relationship of the mandibular first molar to the maxillary first molar and almost always accompanying the problem is the mesial or Class III relation of the mandibular canine to its maxillary counterpart. Although not always in Class III malocclusion have a Class III molar relationship, sometimes the molar relationship could be class or cusp to cusp, whether unilateral or bilateral. ${ }^{2}$

The other dental characteristic is a posterior crossbite, unilateral or bilateral. It might be caused by the mandible is too wide or normal while the maxilla is too narrow or there is a maxillary deficiency indicated by narrow width of the palatal vault. ${ }^{22}$

\section{Facial profile}

Characteristics of Class III malocclusion are an excessive lower face, the prominence of the chin and a concave profile. Profile angle is formed by connecting soft tissue glabella, subnasale, and soft tissue pogonion (Fig. 7). ${ }^{2}$

But not all malocclusion Class III has the same appearance, it depends on the problematic areas. Class III malocclusion caused by mandibular prognathism, is charaterized by a prominent lower third of the face, a steep mandibular plane, flattened labiomentalis sulcus and an acute nasolabial angle. In frontal view, the patients with mandibular excess exhibits a broad lower third 
of the face. In severe cases, lip incompetence may be seen and asymmetry of the lower third of the face often accompanies mandibular excess. The negative overjet does not provide adequate support for either the upper and lower lip (Fig. 8). ${ }^{17}$

Patients with mid-face deficiency have a liitle bit different characteristic appearances. Frequently, the mandible appears prognathic, with an accompanying upward and forward rotation of the mandible and also lead to lip redudancy. Such lip contour is indicate a positive lip step, which is the protrusion of the lower lip in relation with upper lip (Fig. 9). ${ }^{23}$

Maxillary deficiency is variably manifest in infraorbital and paranasal regions, the canine fossae, the malar eminences, and the upper lip.

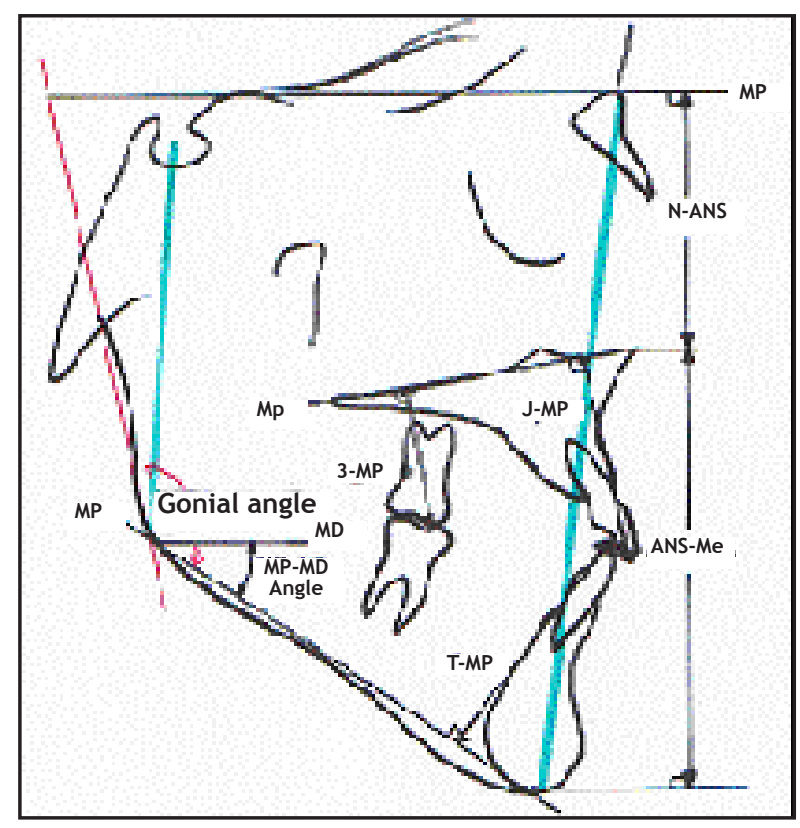

Figure 4. Cephalometri of Class III malocclusion with obtuse gonial angle, greater vertical lower facial height, lower border of the mandible was steeply inclined. ${ }^{12}$

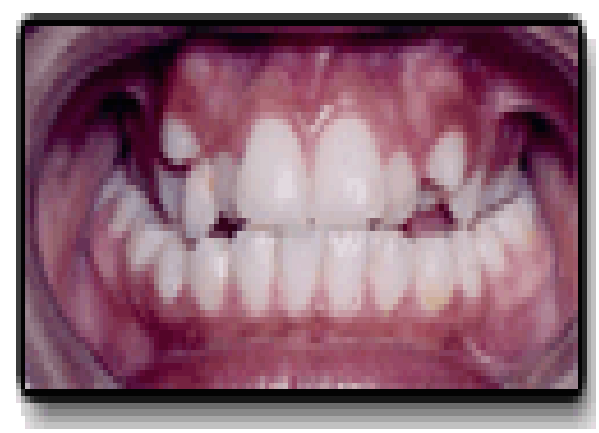

Figure 5. Underdeveloped maxilla because of the ectopic canines and palatal eruption of lateral incisors. ${ }^{20}$

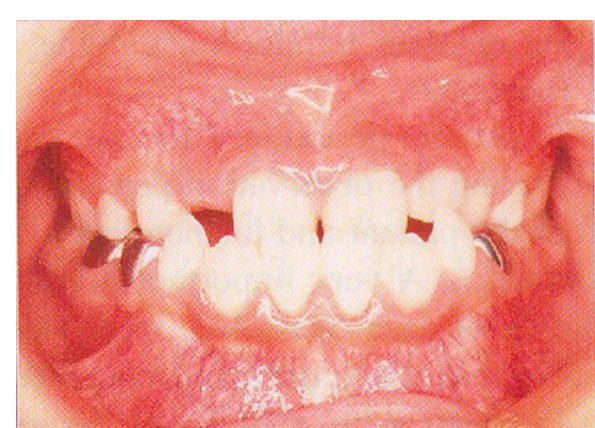

SHORT

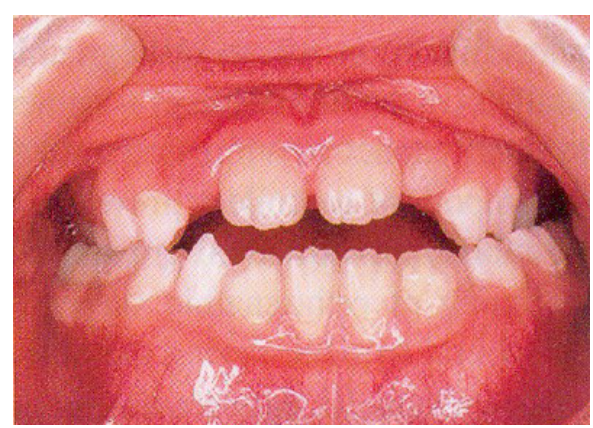

AVERAGE

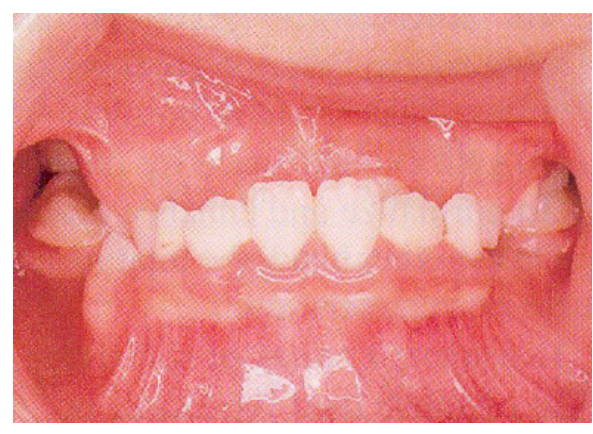

LONG

Figure 6. Occlusion of each skeletal type. ${ }^{11}$

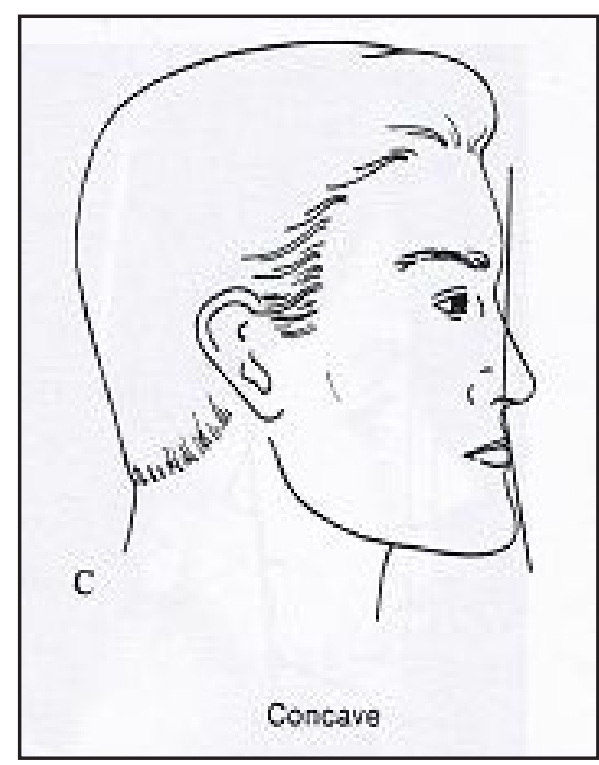

Figure 7. A concave profile. ${ }^{2}$ 

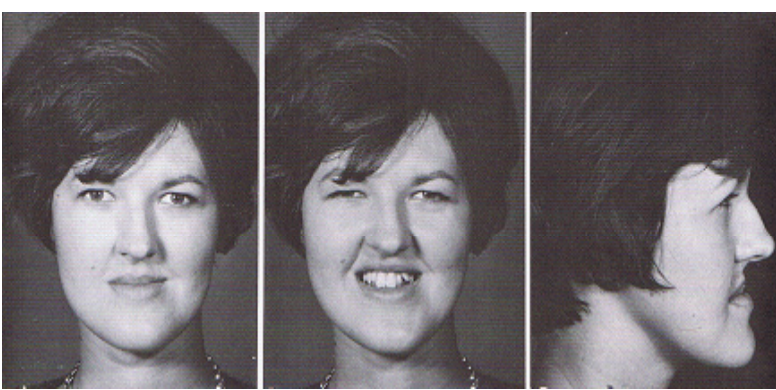

Figure 8. Class III malocclusion caused by mandibular prognathism. ${ }^{17}$

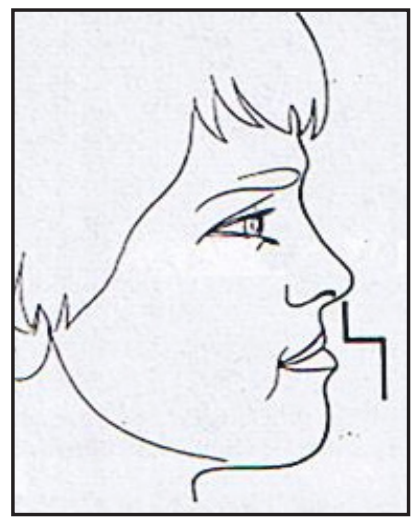

Figure 9. Positive lip step, in Class III malocclusion. ${ }^{23}$

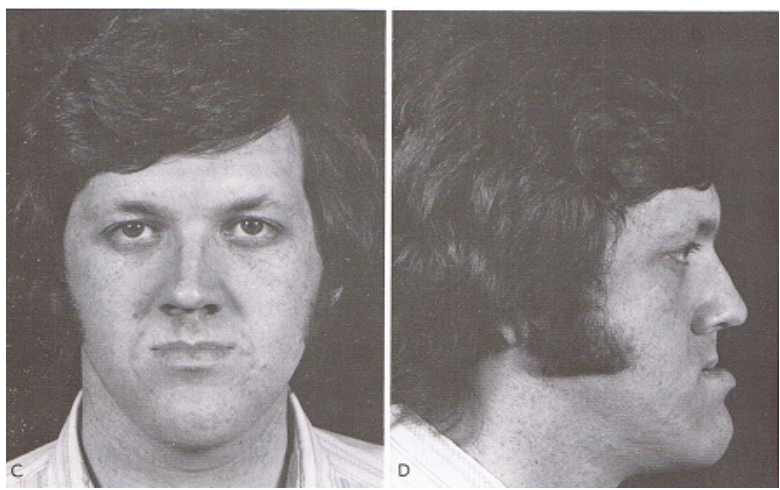

Figure 10. Class III malocclusion caused by maxillary deficiency. ${ }^{12}$

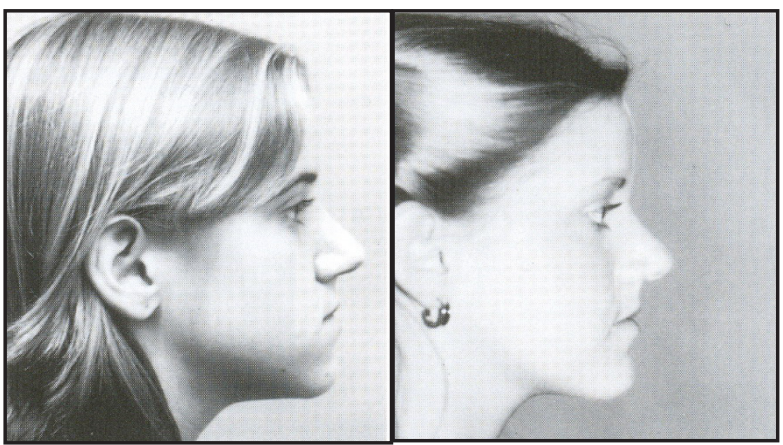

Figure 11. A and B Profile before and after orthodontic camouflage treatment in mild skeletal Class III malocclusion.

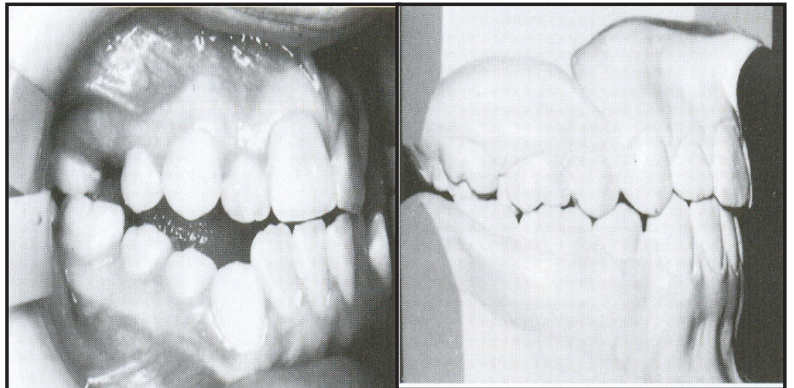

Figure 12. A and B Dental relationship before and after orthodontic camouflage treatment in mild skeletal Class III malocclusion. ${ }^{2}$
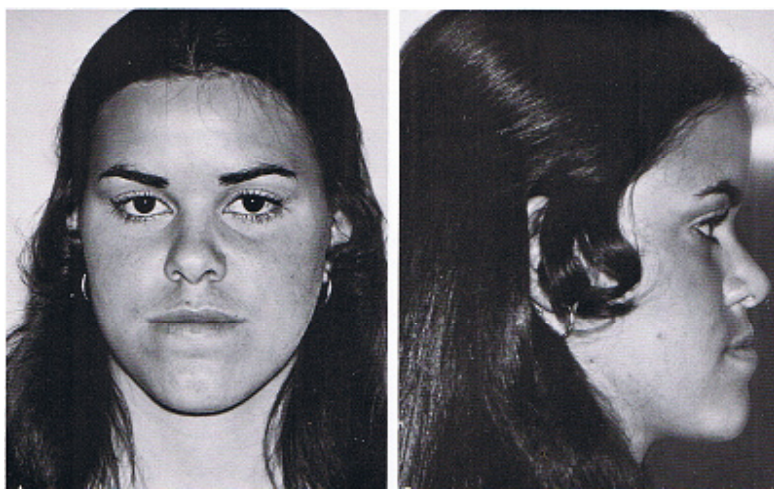

Figure 13. Facial appearance of patient showing severe malocclusion Class III from fontal and lateral aspects. ${ }^{17}$

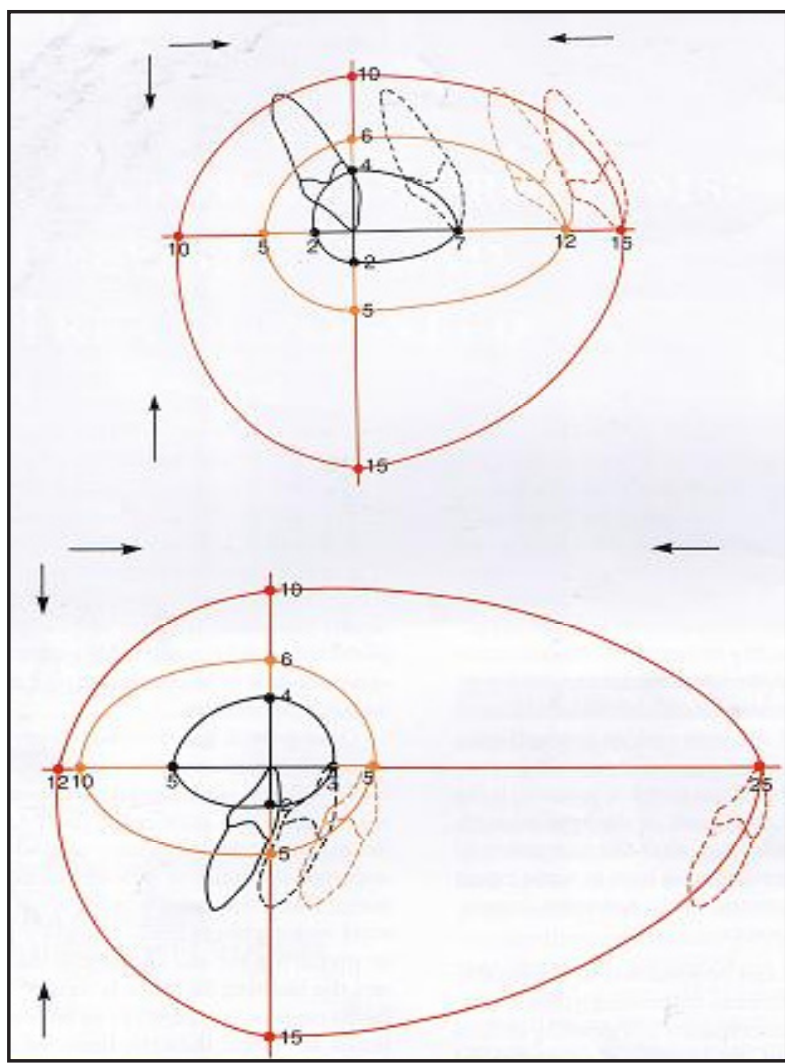

Figure 14. Envelope discrepancy introduced by Proffit and Ackerman. $^{2}$ 
an aobtuse gonial angle and flatness in the paranasal areas are typical findings in maxillary deficiency (Fig.10). ${ }^{12}$

Mandibular overclosure accentuates the midface concavity. In patients with a combination of mandibular excess and maxillary deficiency, these soft tissue contours increase the clinical impression of maxillary deficiency. ${ }^{12}$

\section{Severity of skeletal Class III malocclusion}

Mild to moderate skeletal Class III malocclusion. Profile appearance in a mild to moderate skeletal Class III malocclusion usually looks better. The mandibular plane may not be steep and may not be accompanied by mid-face deficiency so the patient does not have a concave profile. Most often there is no maxillary deficiency, so the facial height looks normal. Anterior facial height and growth pattern are normal in mild skeletal Class III malocclusion. The chin is not too prominent so anteroposterior tooth movement does not affect to the profile. Generally, facial appearance in this patient still looks good (Fig. 11). ${ }^{2}$

In dental relationship, there is no severe crowding, dan the horizontal overlap of the incisor teeth is positive or edge to edge. Discrepancy of maxilla and mandibular from sagital and transvere is not too big. Most often, this mild malocclusion is not accompanied by lateral crossbite, asymmetry of the lower jaw, or skeletal open bite and skeletal deep bite (Fig. 12). ${ }^{2}$

\section{Severe skeletal Class III malocclusion ${ }^{17}$}

Most often in severe Class III malocclusion characterized by an obtuse gonial angle, a steep mandibular plane and a small cranial base angle as seen on the lateral cephalometric film. This malocclusion occurs because of undergrowth of the maxilla, overgrowth of the mandible, or both. Patient with vertical craniofacial growth pattern in Class III malocclusion could be accompanied by anterior open bite and lip incompetence.

The facial appearance typical of malocclusion Class III exhibits a prominence of the chin, sunken in face, the prominence of the lower third of the face. A protrusive lower lip, flattened labiomentalis sulcus and an acute nasolabial angle are typical of this severe malocclusion (Fig. 13). ${ }^{17}$

Therearemanyvariant in dental relationship.
Molar relationship in this malocclusion is the mesial relationship of the mandibular fist molar to the maxillary first molar, unilateral or bilateral but sometimes the molar relationship is cusp to cusp, and the mesial or Class III Angle relation of the mandibular canine to its maxillary counterpart is always accompanying the problem. Most often Class III malocclusion is accompanied by anterior and lateral crossbite. ${ }^{12}$

\section{Treatment planning for skeletal Class III malocclusion \\ Bhalajhi $^{24}$ introduced a flow chart that} describes treatment choices for Class III malocclusion. The choice of treatment depends on age and severity of the malocclusion. Treatment choice for non-growing patient based on the severity of the case. Malocclusion with a mild mandibular prognathism and a moderate overbite can be corrected by dentoalveolar movements without correcting the skeletal discrepancy. But in a patient with severe mandibular prognathism, the orthodontic treatment should be combined with orthognatic surgery (Table 1). ${ }^{24}$

Orthodontic camouflage is built around the idea of displacing the teeth relative to the jaws to compensate for a jaw discrepancy. The goals of camouflage are to obtain satisfactory dental and facial esthetics, along with acceptable dental occlusion and function. This is succesful only if dental and esthetics are both sacrificed to obtain acceptable occlusion..$^{13}$ The method was developed as extraction treatment, followed by othodontic treatment. $^{2}$

Class III camouflage is more difficult, not because the tooth movement is more difficult, but because it is more difficult to obtain acceptable esthetics. ${ }^{13,22}$ Retraction of lower incisors makes the chin more prominent. Even minimal retraction often magnifies the facial esthetic problems associated with Class III malocclusion, so the result of treatment will compromise facial esthetic. In facts, attempts to achieve an absolutely ideal dental occlusion can compromise facial esthetics and may be associated with instability after treatment. In the same way, efforts to achieve the most stable result after orthodontic treatment may necessitate compromises in both occlusal and facial esthetics, whereas positioning the teeth to produce ideal facial 
Figure 15. Flow chart for treatment of Class III malocclusion. ${ }^{24}$

esthetics may result in less than optimal occlusion and stability. ${ }^{2}$

\section{DISCUSSION}

As said by Bhalajhi ${ }^{24}$, treatment of skeletal Class III malocclusion in adults depends on the severity of malocclusion. Orthodontic camouflage treatment usually aimed at compensating for the underlying mild-moderate Class III skeletal discrepancy. But an orthodontist should understand the considerations and the limits of dentoalveolar compensation so the goal of camouflage treatment could be achieved. ${ }^{2}$

Soft tissue contour is the first factor that shoud be considered. Dynamically or statically, the soft tissue contours of the face are determined by three interacting factors: (1) the skeletal foundation, which for the mid and lower face is provided by the jaws; (2) the dental support system; (3) the soft tissue mask, influenced by both the underlying hard tissues and components of the soft tissue itself (nose and chin, lip thickness, lip tonicity). Soft tissue response to change incisor position is sometimes difficult to predict, it may be appropriate to evaluate the esthetic effect of dental changes before treatment. Because the changes of incisors position will affect on lip position, lip tonicity, face and chin). Because the incisors position will affect on lip position, lip tonicity, facial form, chin). ${ }^{25}$

Nasolabial angle is the other thing that should be considered for anteroposterior tooth movement. The nasolabial angle measures the inclinaton of the columella in relation to the upper lip. The nasolabial angle should be in the range $102^{\circ} \pm 8^{\circ}$. This angle can change noticeably with orthodontics and surgical procedures that alter the anteroposterior position or inclination of the maxillary anterior teeth. If the angle is acute, the premaxilla segment can be retracted; if the angle is obtuse, the segment must be protracted to improve facial esthetics. ${ }^{22,26}$

Severe midface deficiency or mandibular prognathism creates unattractive lip positions and may affect throat form. Sometimes followed by lip incompetence and excessive lower facial height. This unesthetic condition can rarely be corrected with orthodontics alone, even if normal overjet and overbite are established. In this condition, orthodontics camouflage is rarely satisfactory, and orthognatic surgery should be considered as 
an alternative. ${ }^{25}$

Labiomental sulcus also important to be noted in Class III malocclusion The labiomentalis sulcus, the fold of the soft tissue between the lower lip and the chin, may var greatly in form and depth and is best describe in terms of its shape curve, which should be a symmetric and relatively shallow $\mathrm{S}$. It is affected by the degree of lip support from incisors and by face height. Upright lower incisors tend to result in a shallow labiomentalis sulcus because of lack of lower lip projection, which explains the flat sulcus commonly seen in Class III patients with incisors compensation. ${ }^{19,22}$

If the treatment outcomes showed a good result for a better facial esthetic, then the camouflage outcome would be satisfactory. But if not, the treatment outcome just only a compromise treatment.

Besides the soft tissue contour, dental relationship must be considered before making a decision. On 1985, Proffit dan Ackerman ${ }^{1,2}$ introduced "envelope of discrepancy". This envelope indicate the limits vary both by the tooth movement (in millimeter), possible changes in incisor position from combined orthopedic and orthodontic treatment in growing individuals are shown by the middle envelope; and the limits of change with combined orthodontic and surgical treatment are shown by the outer envelope, with the ideal positions of upper and lower incisor in the anteroposterior and vertical planes is shown in the centre of the incisor diagrams (Fig. 14).

The limits of orthodontic tooth movement are represented by the inner envelope. ${ }^{2}$ The inner envelope for the upper arch suggests that maxillary incisors can be brought back a maximum of $7 \mathrm{~mm}$ by orthodontic tooth movement alone to correct protrusion but can be moved forward only $2 \mathrm{~mm}$. The limit for retraction is established by the lingual cortical plate; the limit for forward movement is established by the lip. Upper incisors can be extruded $4 \mathrm{~mm}$ and depressed $2 \mathrm{~mm}$.

The middle envelope for the upper and lower arches suggest $5 \mathrm{~mm}$ of growth modification in anteroposterior plane. The outer envelope suggests that $10 \mathrm{~mm}$ is the limit for surgical maxillary advancement or downward movement, although the maxilla can be retracted or move up as much as $15 \mathrm{~mm}$, the mandible can be surgically set back $25 \mathrm{~mm}$ but can be advanced only 12 $\mathrm{mm}$. $^{2,22}$

The envelope discrepancy is merely guidelines and may underestimate or overestimate the possibilities for any given patient. It gives the limitations of orthodontic treatment in terms of whether the occlusion could be corrected, not whether deformity could be camouflage.

Patients with severe crowding would not be a good candidate for camouflage treatment. Because in a patients with severe crowding, the extraction spaces will be required to achieve proper alignment of the incisors. So that the extraction spaces wouldn't be available for controlled anteroposterior displacement. ${ }^{2}$

Anteroposterior tooth movement to compensate for a jaw discrepancy is limited to the width of an extraction space or by the amount expansion that will be tolerated by the periodontium and remain stable. If considerable space is required for relief crowding and for leveling, little may be left for the camouflage of a skeletal discrepancy. ${ }^{2}$ Stability is increasing at risk with anteroposterior expansion of mandibular incisors by more than $2 \mathrm{~mm}$ or transverse expansion of the lower arch more than 4 to $5 \mathrm{~mm} .^{25}$

In Class III malocclusion, problems in transverse dimension can be addressed orthodontically if they result mainly from buccal or lingual tipping of the dentition. A jackscrew to open the midpalatal suture is not applicable in adults and attempts to use this technique may result in subsequent periodontal problems. ${ }^{2}$ In short, it behooves the orthodontist to recognize periodontal susceptibility, control gingival inflamation during orthodontic tooth movement, augment thin gingiva in a preventive fashion when indicated, and move teeth facially less than 2 to 3 $\mathrm{mm}$ in a periodontally resistant individual and not at all in a periodontally susceptible patient. ${ }^{25}$

We should consider the limitation for orthodontic treatment in terms of whether the occlusion could be corrected, not whether the deformity could be camouflaged.

Next consideration is skeletal pattern. Patients with a long face or skeletal open bite pattern are difficult to treat orthodontically. Most orthodontic mechanotherapy extrudes teeth somewhat, and in long face patients is particularly likely to result in downward mandibular rotation 
and further lengthening the face, so the treatment outcome can be unsuccesful. 2,27,28 Extrusive tooth movement also occurs when patient with a short face or skeletal deep bite facial pattern are treated, but generally this is desirable, since extrusion of the posterior teeth and lengthening of the face improve the situation. Downward rotation of the mandible still occurs, but because of the geometric arrangement of the skeletal planes, it is often expressed by the mandible dropping inferiorly more than back, facilitating the possibility of camouflage..$^{2,28}$

The clinician must attempt to control the palatal plane, the occlusal plane, and the Frankfort mandibular plane (FMA). Tweed ${ }^{29}$ considered patients with an FMA angle over $30^{\circ}$ to be difficult to treat. If the FMA is maintained at its pretreatment value, or even closed during treatment, vertical dimension is controlled.

If Class III malocclusion associated with skeletal asymmetry, the camouflage orthodontic treatment can be done only if the chin and external bony contours are not involved. The greater the skeletal involvement, the more likely that surgery will be needed. ${ }^{2}$

\section{CONCLUSION}

The camouflage orthodontic treatment in skeletal Class III malocclusion should consider several things, such as (1) facial profile, include soft tissue contours of the face are determined by the skeletal foundation, the dental support system, the soft tissue mask, influenced by both the underlying hard tissues and components of the soft tissue itself; Nasolabial angle and Labiomentalis sulcus. (2) Dental relationship include the following; The envelope discrepancy is merely a guidelines for tooth movement; Severe crowding would not be a good candidate for camouflage treatment, because the extraction spaces will be required to achieve proper alignment of the incisors. So that the extraction spaces wouldn't be available for controlled anteroposterior displacement and Anteroposterior and transverse teeth movement to compensate for a jaw discrepancy is limited to the width of an extraction space or by the amount expansion that will be tolerated by the periodontium and remain stable. (3) Skeletal pattern; Patients with a long face or skeletal open bite pattern are difficult to treat orthodontically but in patients with a short face generally this is desirable, since extrusion of the posterior teeth and lengthening of the face improve the facial esthetic; Patients with an FMA angle over $30^{\circ}$ to be difficult to treat and Skeletal asymmetry, the camouflage orthodontic treatment can be done only if the chin and external bony contours are not involved.

Although we have considerations for camouflage orthodontic treatment in class III malocclusion, there are many individual factors that would make an unsuccessful camouflage outcome. It is wise, if we choose the best plan focuses on patient's more important ones, compromising the less important problem in favor of the more important ones.

\section{REFERENCES}

1. Stellzig-Eisenhauer A, Lux CJ, Schuster G. Treatment decision in adult patients with Class III malocclusion, orthodontic therapy or orthognatic surgery. Am J Orthod Dentofac Orthop 2002;122:27-38.

2. Proffit WR, Fields HW, Ackerman JL. Contemporary orthodontics. $3^{\text {rd }}$ ed. St Louis: Mosby Year Book Inc.; 2000. p. 628-34.

3. Rabie ABM, Gu Yan. Diagnostic criteria for pseudo Class III malocclusion. Am J Orthod Dentofac Orthop 2000;117:1-9.

4. Campbell PM. The dilemma of Class III treatment: early or late. Angle Orthod 1983; 53:175-91.

5. McNamara JA. An orthopedic approach to the treatment of Class III malocclusion in young patient. J Clin Orthod 1987;21:598-608.

6. Nanda R. Biomechanical and clinical consideration of a modified protraction headgear. Am J Orthod 1980;78:125-39.

7. Rakosi T, Sichilli W. Class III anomalies: A coordinated approach to skeletal, dental and soft tissue problems. J Oral Surg 1981;39:86070.

8. Bishara SE. Textbook of orthodontics. Philadelphia: W.B. Saunders Co.; 2001. p. 375411.

9. Janson G, Souza JEP, Alves FA, et al. Extreme dentoalveolar compensation in the treatment of Class III malocclusion. Am J Orthod Dentofac 
Orthop 2005;128:787-94.

10. Jacobson A, Evans WG, Preston CB, Sadowsky PL. Mandibular prognathism. Am J Orthod Dentofac Orthop 1974;66(2):140-71.

11. Sugawara J. Clinical pratice guidelines for developing Class III malocclusion. In: Nanda R. Biomechanics and esthetic strategies in clinical orthodontics. St. Louis: Elsevier Saunders; 2005. p. 211-77.

12. Bell WH, Proffit WR, White RP. Surgical correction of dentofacial deformities Vol 1. Philadelphia: W.B. Saunders Co.; 1980. p. 44357.

13. Bailey LJ, Sarver DM, Turvey TA, Proffit WR. Class III problems. In: Proffit WR, White RP, Sarver DM. Contemporary treatment of dentofacial deformity. St. Louis: Mosby Year Book; 2003. p. 507-27.

14. Guyer, EC, Ellis EE, MCNamara JA, Behrents RG. Components of Class III malocclusion in juveniles and adolescents. Angle Orthod 1986; 56:7-30.

15. [cited 2007 Sep 26] Avalaible from: http:// www.harmonorthodontics.com/case.htm 26 Sep 2007.

16. Sanborn RT. Differences between the facial skeletal patterns of Class III malocclusion and normal occlusion. Angle Orthod 1955;25:20822.

17. Bell WH, Proffit WR, White RP. Surgical correction of dentofacial deformities. Philadelphia: W.B. Saunders Co.; 1980. p. 84449.

18. Singh GD, McNamara JA, Lozanoff S. Finite element analysis of the cranial base in subjects with Class III malocclusion. Br J Orthod 1997; 24:103-112.
19. Sarver DM. Esthetics orthodontics and orthognatic surgery. St. Louis: Mosby Year Book Inc.; 1998. p. 9-59.

20. [cited 2007 Sep 26] Avalaible from: http:// www. petalumaorthodontics.com/treaments/ crossbite.html 26 sep 2007.

21. Ishikawa $\mathrm{H}$, Nakamura $\mathrm{S}$, Iwasaki $\mathrm{H}$, et al. Dentoalveolar compensation in negative overjet cases. Angle Orthod 2000;70(2):1458.

22. Graber TM, Vanarsdall RL. Orthodontics. Current principles and techniques. $2^{\text {nd }}$ ed. St. Louis: Mosby Year Book Inc.; 1994. p. 80-94.

23. Rakosi T, Jonas I, Graber TM. Color atlas of dental medicine: orthodontic diagnosis. New York: Thieme Medical Publisher Inc.; 1993. p. 112-15.

24. Bhalajhi SI. Orthodontics: the art and science. $3^{\text {rd }}$ ed. New Delhi: Arya (medi) Publishing House; 2003. p. 407-14.

25. Ackerman JL, Proffit WR. Soft tissue limitation in orthodontics: treatment planning guidelines. Angle Orthod 1997;67(5):327-36.

26. Graber TM, Rakosi T, Petrovic AG. Dentofacial orthopedics with functional appliance. $2^{\text {nd }}$ ed. St. Louis: Mosby; 1997. p. 463-80.

27. Nanda SK. Patterns of vertical growth in the face. Am J Orthod Dentofac Orthop 1988; 93:103-16.

28. Sato S. Case report: developmental characterization of skeletal Class III malocclusion. Angle Orthod 1994;64(2):10511.

29. Bilodeau JE. Vertical considerations in diagnosis and treatment. A surgical orthodontic case report. Am J Orthod Dentofac Orthop 1995; 107:91-100. 